\title{
Sustained tumour eradication after induced caspase-3 activation and synchronous tumour apoptosis requires an intact host immune response
}

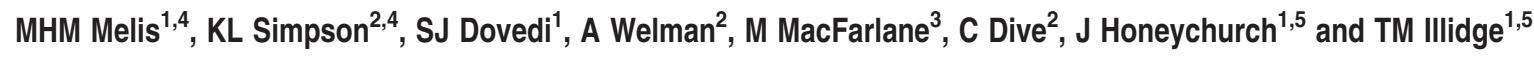

Effective anticancer treatments often result in the induction of large amounts of tumour cell death. In vivo, such dying tumour cells are a potential source of antigens for T-cell stimulation. Although apoptosis is generally considered nonimmunogenic, recent evidence suggests that some anticancer therapies that induce apoptosis can elicit antitumour immune responses. Here, a doxycycline-inducible, constitutively active caspase-3 ('death switch') was constructed in a murine tumour model to explore the impact of the host immune response to rapid, synchronous and substantial tumour cell apoptosis. In vitro, up to $80 \%$ of tumour cells underwent apoptotic cell death within $24 \mathrm{~h}$ and death was accompanied by the release of potential 'danger signal' molecules HMGB1 and HSP90. In vivo, death switch induction provoked rapid, pronounced tumour regression in immune-competent and immune-deficient mice, but sustained tumour eradication was observed only in immune-competent mice. Moreover, the majority of mice that were tumour free after death switch induction were protected from further tumour rechallenge. In addition, long-term remission after induction of the death switch was completely abrogated following depletion of CD8 T cells. These data suggest that sustained tumour eradication after substantial tumour apoptosis requires an antitumour host immune response that prevents tumour relapse. In many patients, cancer therapies produce encouraging initial responses that are only short lived. These results provide new insights that may have important implications for further development of strategies that result in long-term tumour clearance after initially effective anticancer treatment.

Cell Death and Differentiation (2013) 20, 765-773; doi:10.1038/cdd.2013.8; published online 15 February 2013

Cancer cells dying as a consequence of cytoreductive therapies such as chemotherapy or radiation are a potential source of antigen for priming a cognate immune response. Theoretically, induction of an effective antitumour immune response could eradicate minimal residual disease and maintain clinical remission. However, appropriate immune recognition of dying tumour cells is a major obstacle to eliciting such an outcome. The immune response to physiological, programmed cell death (PCD) is carefully regulated to ensure that normal tissue homeostasis does not produce adverse autoimmune reactions. Thus, apoptosis is considered to be immunologically ignored or functions to suppress immune activation via the induction of tolerance, with apoptotic bodies rapidly cleared by phagocytes. ${ }^{1}$ In contrast, necrotic cell death is associated with loss of plasma membrane integrity, facilitating release of danger signals ${ }^{2}$ or damage-associated molecular patterns ${ }^{3}$ (DAMPs) including uric acid, ${ }^{4}$ heat shock proteins (HSPs) ${ }^{5}$ and mitochondrial constituents ${ }^{6}$ that serve as endogenous immune adjuvants. DAMPs released from dying cells can stimulate an effective immune response against cellular antigen, in much the same way as pathogenic signals arising from infection. Cancer cells undergoing apoptosis as a result of cytotoxic therapy are thought to be perceived in the same manner as physiological cell death and therefore unable to prime effective, adaptive immunity.

Recent data have however challenged the proposed immunological distinction between apoptosis and necrosis as an oversimplification, and it is clear that various subtly different subroutines exist with distinct molecular characteristics. ${ }^{7}$ Although many of these pathways are known to be induced in tumour cells following antineoplastic therapy, ${ }^{8}$ the immunological consequences are only just beginning to be understood or remain largely unknown., ${ }^{9,10}$ Importantly, tumour cells treated with certain chemotherapeutic agents such as anthracyclines have been shown to undergo a more immunogenic form of apoptosis ${ }^{11}$ characterised by the expression of calreticulin, ${ }^{12}$ high-mobility group box 1 $(\mathrm{HMGB} 1)^{13}$ and adenosine triphosphate (ATP). ${ }^{10}$ Together,

\footnotetext{
${ }^{1}$ Targeted Therapy, Institute of Cancer Sciences, Manchester Academic Health Science Centre, University of Manchester, Manchester, UK; ${ }^{2}$ Clinical and Experimental Pharmacology Group, Paterson Institute for Cancer Research, Manchester University, Manchester Cancer Research Centre, Manchester Academic Health Sciences Centres, Manchester, UK and ${ }^{3}$ MRC Toxicology Unit, University of Leicester, Leicester, UK

${ }^{*}$ Corresponding author: TM Illidge, Institute of Cancer Sciences, Manchester Academic Health Science Centre, University of Manchester, Manchester, M20 4BX, UK. Tel: +44 161446 8110; Fax: +44 161446 3296; E-mail: tmi@manchester.ac.uk

${ }^{4}$ These authors are joint first authors.

${ }^{5}$ These authors are joint senior authors.

Keywords: apoptosis; cancer; immunogenicity

Abbreviations: AxV, annexin V; DAMP, damage-associated molecular pattern; DC, dendritic cell; Dox, doxycycline; HMGB1, high-mobility group box 1; HSP, heat shock protein; PCD, programmed cell death; PI, propidium iodide; Q-VD-OPh, N-(2-quinolyl) valyl-aspartyl-(2,6-difluorophenoxy) methyl ketone; RevC3, reverse caspase-3; rtTA, reverse transcriptional transactivator; SRB, sulforhodamine $B$

Received 06.8.12; revised 20.12.12; accepted 07.1.13; Edited by RA Knight; published online 15.2.13
} 
these promote the acquisition of apoptotic tumour cells by dendritic cells (DCs); enhance Toll-like receptor 4 (TLR4)dependent antigen processing and cross-presentation by DCs; and stimulate production of interleukin- $1 \beta$ that is required for the effective priming of tumour-specific CD8 T cells. ${ }^{10,12,13}$ Radiation therapy has also been shown to induce an immunogenic form of apoptosis. ${ }^{14}$

In view of these recent studies regarding immunogenic apoptosis, coupled with the observation that necrotic cell death may not always stimulate immunity, ${ }^{15}$ the current understanding regarding the immunogenicity of tumour cell death mechanisms needs to be reassessed. In order to address questions regarding the immunogenic potential of malignant cells dying in vivo, appropriate model systems with tightly controlled induction of specific cell death mechanisms are highly desirable as they facilitate molecular dissection of the host immune response. Here, we report the development of a syngeneic murine tumour model transfected with a tetracycline-regulated reverse transcriptional transactivator (rtTA), ${ }^{16}$ facilitating the conditional induction of constitutively active caspase- 3 termed the 'death switch'. Induction of this 'death switch' results in rapid, synchronous apoptosis both in vitro and in vivo. We demonstrate that activation of this 'death switch' results in regression of tumours and have identified an important immunological component required for long-term clearance of established tumour cells dying in vivo by apoptosis.

\section{Results}

Generation of caspase-3 'death switch' murine tumour lines. The murine B16 melanoma cell lines (B16F10, B16F1 and B16ova) have been well characterised in tumour immunology studies and are therefore good candidates to develop the caspase-3-dependent 'death switch' model. To overcome the requirement for proteolytic activation of caspase-3, the death switch system exploits a constitutively active, reverse caspase-3 (revC3), which mimics the processed molecule. ${ }^{17}$ The principle for the two-step protocol used to generate 'death switch' lines is shown in Figure 1a (based on Welman et al. ${ }^{16}$ ). Tet-on cell line variants transfected with module 1 were selected for transfection with a second construct containing revC3 (described in Srinivasula et al. ${ }^{17}$ ) sub-cloned into the pTRE2hyg vector. Thus, lines were established in which activation of the inducible promoter and expression of revC3 is conditionally triggered by the tetracycline analogue doxycycline (dox), resulting in apoptosis: the 'death switch'. For simplicity, data regarding generation and functional screening of the B16ova revC3 cell line will be shown, hereafter referred to as death switch cells. However, clones derived from all cell lines showed similar behaviour.

Death switch clones were screened and selected on their ability to exhibit dox-induced biochemical and morphological features of apoptosis. Clones showed comparable growth kinetics to the untransfected parental cell line in vitro, confirmed using the colorimetric sulforhodamine B (SRB) assay (Supplementary Figure 1). Flow cytometric analysis of annexin $\mathrm{V} /$ propidium iodide (AxV/PI) staining was performed to measure the amount of death. Clones expressing revC3 underwent cell death within 6-12 h after induction of the death switch, rapidly progressing towards secondary necrosis (Supplementary Figure 2). A highly significant increase in AxV/PI positivity was observed in the death switch clones 12-24 $\mathrm{h}$ after addition of dox compared with vehicle controls or untransfected parental cells (Supplementary Figure 2 and Figure $\left.1 b ;{ }^{* \star} P<0.01\right)$. This was mirrored by changes in size/ granularity consistent with apoptotic death (Supplementary Figure 3). Addition of the pan-caspase inhibitor Q-VD-OPh ( $N$-(2-quinolyl) valyl-aspartyl-(2,6-difluorophenoxy) methyl ketone) before dox resulted in a significant reduction in AxV/PI positivity (Figure $1 \mathrm{~b} ;{ }^{* *} P<0.01$ ) and size/granularity changes (Supplementary Figure 3 ), confirming that dox-induced death was caspase dependent. In the presence of dox, a sub-G1 population, indicative of DNA fragmentation occurring during apoptosis, was observed using hypotonic PI staining, which was also inhibited by Q-VD-OPh (Supplementary Figure 4). Both cleaved caspase-3 and its downstream target cleaved PARP could be detected by western blotting following death switch induction, which could be blocked by addition of Q-VD-OPh (Figure 1c). Caspase activity was undetectable in parental cells either in the presence or absence of dox, and in untreated death switch cells. Finally, morphological assessment was performed using transmission electron microscopy. Cells dying after activation of the death switch uniformly displayed classical features of apoptosis including chromatin condensation and blebbing (Figure 1d). Together, these data demonstrate that induction of the death switch results in caspase-dependent apoptosis as determined using a range of biochemical and morphological assessments.

Death switch-induced apoptotic cell death is associated with the release of HMGB1 and HSP90. Conventionally, apoptosis has been considered a 'contained form' of cell death that does not stimulate adaptive immunity. However, a form of immunogenic apoptosis has recently been described, defined by expression of key DAMPs both early on in the cell death pathway and at later stages, as apoptotic cells progress towards secondary necrosis. ${ }^{11} \mathrm{We}$ therefore investigated whether apoptosis induced by activation of the death switch was associated with expression of constituents capable of functioning as immune adjuvants. Both HMGB1 and HSP9O could be detected in culture supernatant following treatment of death switch cells with dox, but not vehicle control, and this release could be inhibited by addition of Q-VD-OPh, indicating the requirement for caspase activation in this process (Figure 2a). Release was not detectable in the supernatants of parental cells treated with dox (not shown). Release of HMGB1 and HSP90 occurred from $12 \mathrm{~h}$ after induction of the death switch (Figure $2 \mathrm{~b}$ ). To determine whether DAMP release was an early phenomenon during cell death or occurred later in the pathway as cells progressed towards a necrotic phenotype, death switch cells were sorted according to membrane integrity (PI positivity) $12 \mathrm{~h}$ after treatment with dox. PI-positive and $\mathrm{PI}$ negative fractions were cultured for a further $6 \mathrm{~h}$ and supernatants probed for HMGB1. Interestingly, HMGB1 was only detectable in the PI-negative fraction, suggesting that release of DAMP had already occurred by the time cells 
a

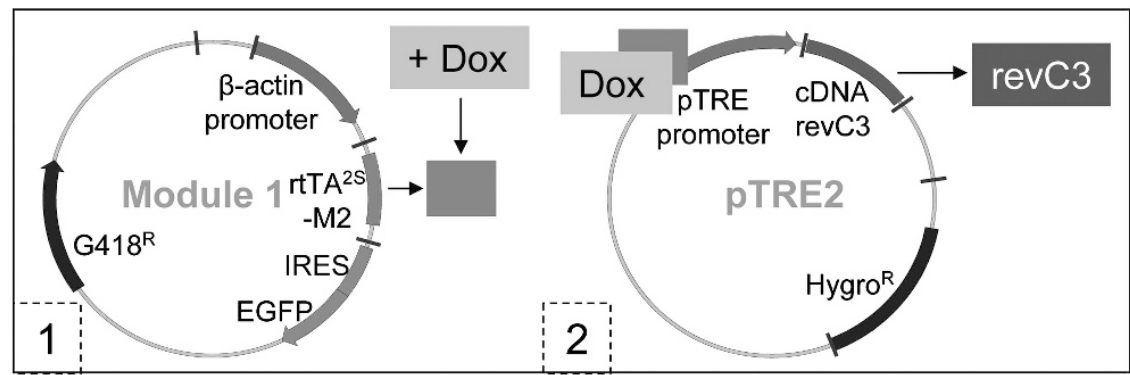

b

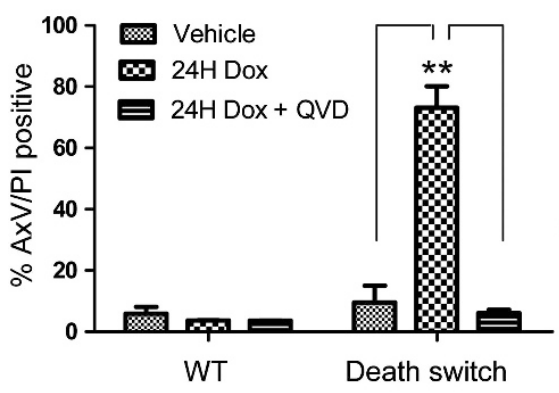

d

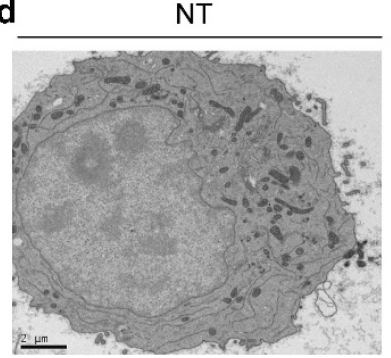

C

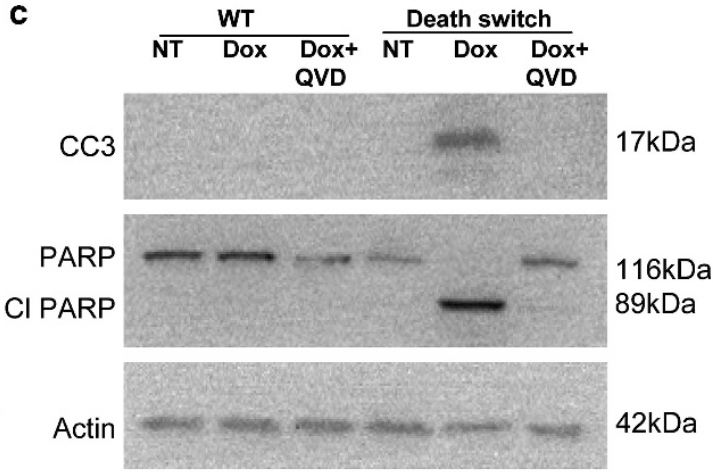

Death switch

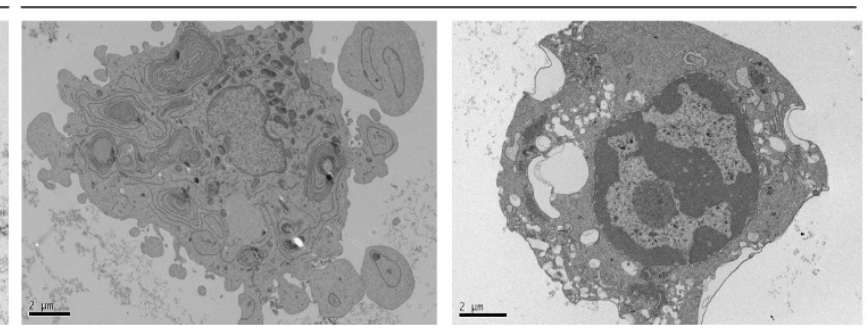

Figure 1 Characterisation of the doxycycline-regulated caspase-3 death switch model in vitro. B16ova RevC3 or parental nontransfected cells were treated with dox $(2 \mu \mathrm{g} / \mathrm{ml})$ or vehicle control and assessed after $24 \mathrm{~h}$ for induction of cell death. (a) Schematic representation of the two-step transfection process for generation of doxregulated caspase-3 death switch models. (b) Quantitation of annexin V/propidium iodide staining in the presence or absence of the pan-caspase inhibitor Q-VD-OPh (20 $\mu \mathrm{M})$. Data represent mean \pm S.E.M. from three independent experiments. ${ }^{* *} P<0.01$. (c) Immunoblot analysis of cleaved caspase-3 and cleaved PARP in parental (WT) or death switch transfected cells following treatment with dox in the presence or absence of Q-VD-OPh. $\beta$-Actin is included as a loading control. (d) Transmission electron microscopy images of death switch cells untreated (NT) or treated with dox. Bars $=2 \mu \mathrm{m}$

reached later-stage necrosis (Supplementary Figure 5). Furthermore, release of potential danger signals correlated with the degree of cell death. This relationship was explored by culturing death switch cells with parental cells at varying ratios. There was a clear increase in release of HMGB1 and HSP90 correlating with the degree of cell death as the ratio of death switch cells within the co-culture increased $\left(R^{2}=0.93\right.$, ${ }^{\star \star} P<0.01$; Figure 2c). This suggests that release of endogenous danger signals is a consequence of death switch induction rather than a bystander response of viable cells.

In addition to release of DAMPs, ectopic expression of calreticulin is reported to be an early event during immunogenic apoptosis. ${ }^{18}$ We therefore determined whether translocation of calreticulin to the cell surface occurred after activation of the death switch, using immunofluorescence staining. Death switch cells in the absence of dox were used to determine baseline expression; addition of the protein phosphatase 1 inhibitor, calyculin A, was used as a positive control. ${ }^{12}$ No increase in ectopic calreticulin was observed in the presence of dox, but enhanced expression could be detected following incubation with calyculin A (Figure 2d). Together, these data indicate that apoptosis arising from death switch induction is accompanied by release of potential DAMPs as cells progress towards secondary necrosis, but lacks certain key features of immunogenic apoptosis.

Tumour cells dying after death switch induction are recognised and engulfed by DCs. We then assessed whether apoptotic cells generated by the death switch were recognised and engulfed by DCs. Using primary bone marrow-derived murine DCs, the degree of uptake was determined using a well-established flow cytometric method. Death switch cells were labelled with the lipophilic membrane dye PKH26, exposed to dox for $24 \mathrm{~h}$ to induce apoptotic cell death and co-cultured with primary DCs. Death switch cells in the absence of dox were used as a control. Uptake was assessed by flow cytometry using CD11c staining to identify 

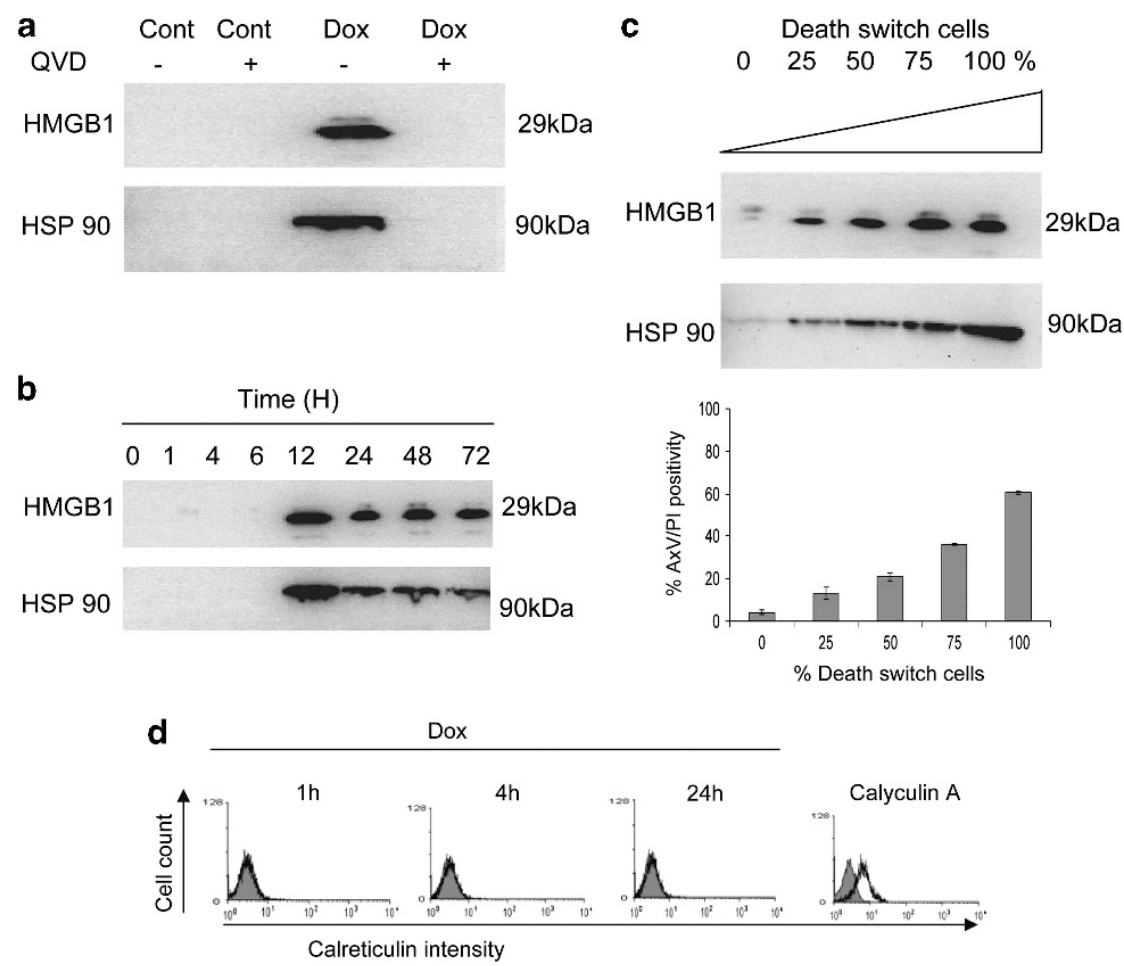

Figure 2 Caspase-3-induced cell death leads to expression of DAMP. Death switch cells were treated with dox (2 $\mu \mathrm{g} / \mathrm{ml})$ or vehicle control (Cont) and assessed over the indicated time course for expression or release of DAMP. Immunoblot analysis of HSP90 and HMGB1 released in to culture supernatant (a) $24 \mathrm{~h}$ after treatment with dox in the presence or absence of the pan-caspase inhibitor Q-VD-OPh $(20 \mu \mathrm{M})$ and $(\mathbf{b})$ at time points up to $72 \mathrm{~h}$. (c) DAMP release corresponds to the degree of cell death (annexin V/ propidium iodide positivity). Histogram data represent mean \pm S.E.M. from three independent experiments. (d) Surface expression of calreticulin on death switch cells was analysed by flow cytometry at various time points after dox treatment as indicated. Calyculin A was used as a positive control. Images are representative of at least three independent experiments

DCs with dual-labelled $\left(\mathrm{PKH} 26^{+} \mathrm{CD} 11 \mathrm{c}^{+}\right)$cells representing tumour cells engulfed by DCs (Figure 3a). Induction of the death switch resulted in significant uptake of tumour cells compared with untreated controls $\left({ }^{*} P<0.05\right)$, which could be inhibited by addition of cytochalsin $\mathrm{D}$, an actin polymerisation inhibitor, which inhibits DC phagocytosis (Figure 3b).

Induction of the death switch in vivo results in tumour regression. We next confirmed that the death switch could be activated in vivo following administration of dox. Death switch clones showed normal growth in vivo compared with the parental line when implanted s.c. (Supplementary Figure 6). Animals were given dox when the mean tumour volume reached $\sim 350 \mathrm{~mm}^{3}$. Treatment with a single dose of dox was sufficient to induce apoptosis in tumours within $24 \mathrm{~h}$ as determined by immunohistochemistry for cleaved PARP (Figure $4 \mathrm{a}$ ). At this time point, over $85 \%$ of tumour sections showed staining of grade $\geq 2$ for cleaved PARP, compared with only $19 \%$ of vehicle-treated controls, which predominantly showed staining of grade $0-1$ (Supplementary Table 1). Corresponding with this increase in cleaved PARP, treatment with dox also led to a significant reduction in tumour volume in death switch but not parental cells (Figure 4b); however, death switch tumours recovered from this initial regression and rapidly re-grew (data not shown). To determine whether a more prolonged application of dox would enhance tumour growth delay, animals inoculated with death switch cells as before were given dox daily for 7 days. Intriguingly, following this extended dosing regimen, tumour regression was maintained relative to controls even upon cessation of dox administration (Figures $4 \mathrm{c}$ and d), and over $40 \%$ of animals showed a significant $\left({ }^{\star *} P<0.01\right)$ long-term ( $>80$ days) increase in survival (Figure $4 \mathrm{e}$ ). This observation prompted us to investigate whether durable tumour remissions were associated with the induction of a host immune response. To address this question, mice that had previously rejected their primary tumours were rechallenged with viable tumour cells, in the absence of further dox administration. Notably, long-term survivors demonstrated a statistically significant $\left({ }^{* *} P<0.01\right)$ improvement in overall survival following rechallenge as compared with control, naive mice (Figure 4f), with the majority able to completely reject secondary tumour, suggesting that induction of synchronous apoptosis can prime adaptive immunity.

Sustained tumour control in response to death switch-induced apoptosis requires a host adaptive immune response. To examine this hypothesis, we determined whether induction of the death switch in immunocompromised animals would lead to long-term clearance of tumour. Parallel cohorts of C57BI/6 and NOD/SCID mice were inoculated with death switch cells and treated as before. Interestingly, although initial tumour regression was observed in both cohorts (Figure 5a), sustained tumour 
a
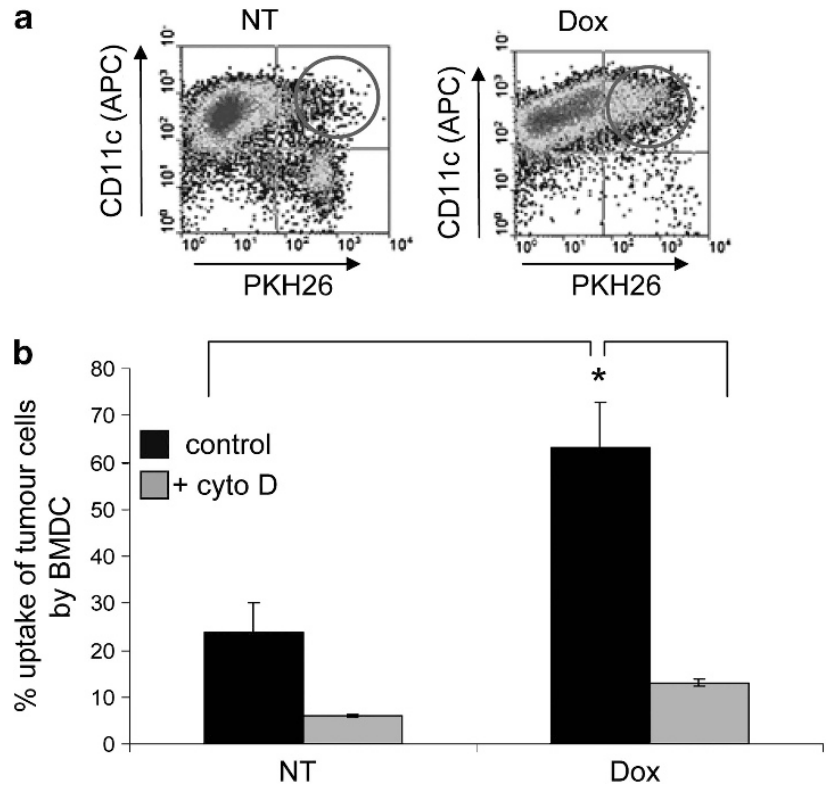

Figure 3 Functional consequences of caspase-3 death switch-induced cell death. Uptake of death switch cells by murine BMDCs was assessed $24 \mathrm{~h}$ after induction of the death switch with dox $(2 \mu \mathrm{g} / \mathrm{ml})$ or in the absence of death switch induction (NT). (a) Representative flow cytometry dot plot indicating uptake (CD11Cpositive/PKH26-positive cells; circled) and (b) quantification of cell uptake in the presence or absence of cytochalasin $D($ cyto D). Data represent mean \pm S.E.M. from three independent experiments. ${ }^{*} P<0.05$

remission and long-term survival $\left({ }^{* \star} P<0.01\right)$ were only observed in immune-competent animals (Figure $5 \mathrm{~b}$ ). In light of these observations, we postulated that durable tumour clearance may be dependent upon an anti-tumour CD8 T-cell response. To confirm the importance of CD8 $T$ cells to sustained survival, death switch induction was conducted in the presence of CD8-depleting mAb. Depletion of CD8positive cells from the peripheral blood was confirmed by flow cytometry (Figure 6a). Importantly, after depletion of CD8 $T$ cells, no significant difference was observed between control cohorts and those receiving dox either in terms of tumour growth delay (Figure $6 b$ ) or overall survival (Figure 6c; $P=0.3$ ). However, a significant effect on tumour growth and survival was observed in 'non-depleted' mice $\left({ }^{* *} P<0.01\right.$; Figures $6 \mathrm{~b}$ and $\left.\mathrm{c}\right)$. Thus, contrary to expectation, it appears that induction of apoptotic cell death in established tumours is sufficient to lead to eradication of disease via CD8 $\mathrm{T}$ cell-dependent immunity.

\section{Discussion}

Here we have utilised a tetracycline-inducible gene expression system to enable the conditional induction of caspase-3dependent cell death, termed the 'death switch', in syngeneic murine melanoma lines. Caspase- 3 was the molecular target of choice given that it plays a central role in executing apoptotic cell death triggered through both extrinsic and intrinsic signalling cascades, ${ }^{19}$ and has previously been shown to be an effective target for chemically induced dimerisation cell elimination strategies. ${ }^{20}$ Activation of the death switch results in rapid, synchronous apoptosis displaying many of the classical biochemical and morphological features associated with caspase-3 activity both in vitro and in vivo. In addition, cell death is accompanied by the release of intracellular constituents such as HMGB1 and HSP90. Induction of the death switch in vivo results in eradication of tumour and enhanced survival. Unexpectedly, clearance of tumour appears to require an adaptive immunological component, as tumours rapidly regrow in immunodeficient or CD8 T cell-depleted animals. These observations highlight the possibility that apoptotic cell death in established tumour may not be immunologically silent.

Our data demonstrate that induction of revC3 initiates tumour apoptosis accompanied by release of intracellular contents including HMGB1 and HSP90. This is in contrast to published data showing that release of the chromatin-binding protein HMGB1 is an exclusive consequence of necrosis, with HMGB1 retained in apoptotic cells, even those progressing to secondary necrosis. ${ }^{21}$ Likewise, liberation of HSP has been identified as a property limited to necrotic cell death. ${ }^{22}$ The differential release of intracellular contents has been used to define the immunological properties of necrotic and apoptotic cell death as pro-inflammatory and tolerogenic/silent, respectively. However, the legitimacy of this distinction is increasingly questioned. A variety of stimuli have now been shown to trigger the release of HMGB1 from apoptotic cancer cells as they progress towards secondary necrosis ${ }^{13,23}$ and this can lead to enhanced immunogenicity. ${ }^{13}$ In addition, apoptotic cells may release extracellular ATP ${ }^{24}$ along with other potentially inflammatory molecules such as DNA. ${ }^{25}$ Data obtained using the death switch model are compatible with the notion that intracellular contents can be released as cells progress through the apoptotic pathway. Interestingly, in our model it appears that DAMPs are released shortly after membrane integrity is lost. Cells sorted on the basis of $\mathrm{PI}$ inclusion and subsequently screened for HMGB1 release demonstrate that it is the $\mathrm{PI}$-negative/low population that predominantly releases HMGB1. An important caveat to this observation is that cells continue to die once selected and that a proportion of PI-negative cells become PI positive during the subsequent culture period used to detect HMGB1. However, HMGB1 release could not be detected from PI-positive selected cells, suggesting that release occurs early on during the progression to secondary necrosis rather than at later stages of terminal necrosis. The extent to which DAMP release enhances immunogenicity remains unclear. Although a broad range of cytotoxic drugs are able to stimulate release of HMGB1 and ATP from apoptotic tumour cells, ${ }^{23,24}$ only a limited few are able to induce immunogenic cell death. ${ }^{12}$ Translocation of selective intracellular contents to the extracellular milieu as apoptotic cells progress towards late apoptosis/secondary necrosis may be insufficient to enhance immunogenicity. First, cooperative signalling via expression of additional determinants such as ectopic calreticulin ${ }^{12}$ that are not expressed during all forms of apoptosis may be required. Alternatively, post-translational modification of proteins released during certain cell death subroutines may affect their functional activity. For example, reactive oxygen species derived from mitochondria as a consequence of caspase activation can oxidise cysteine residues within HMGB1, rendering the protein tolerogenic rather than immunogenic. $^{26}$ Moreover, oxidation of HMGB1 affects 

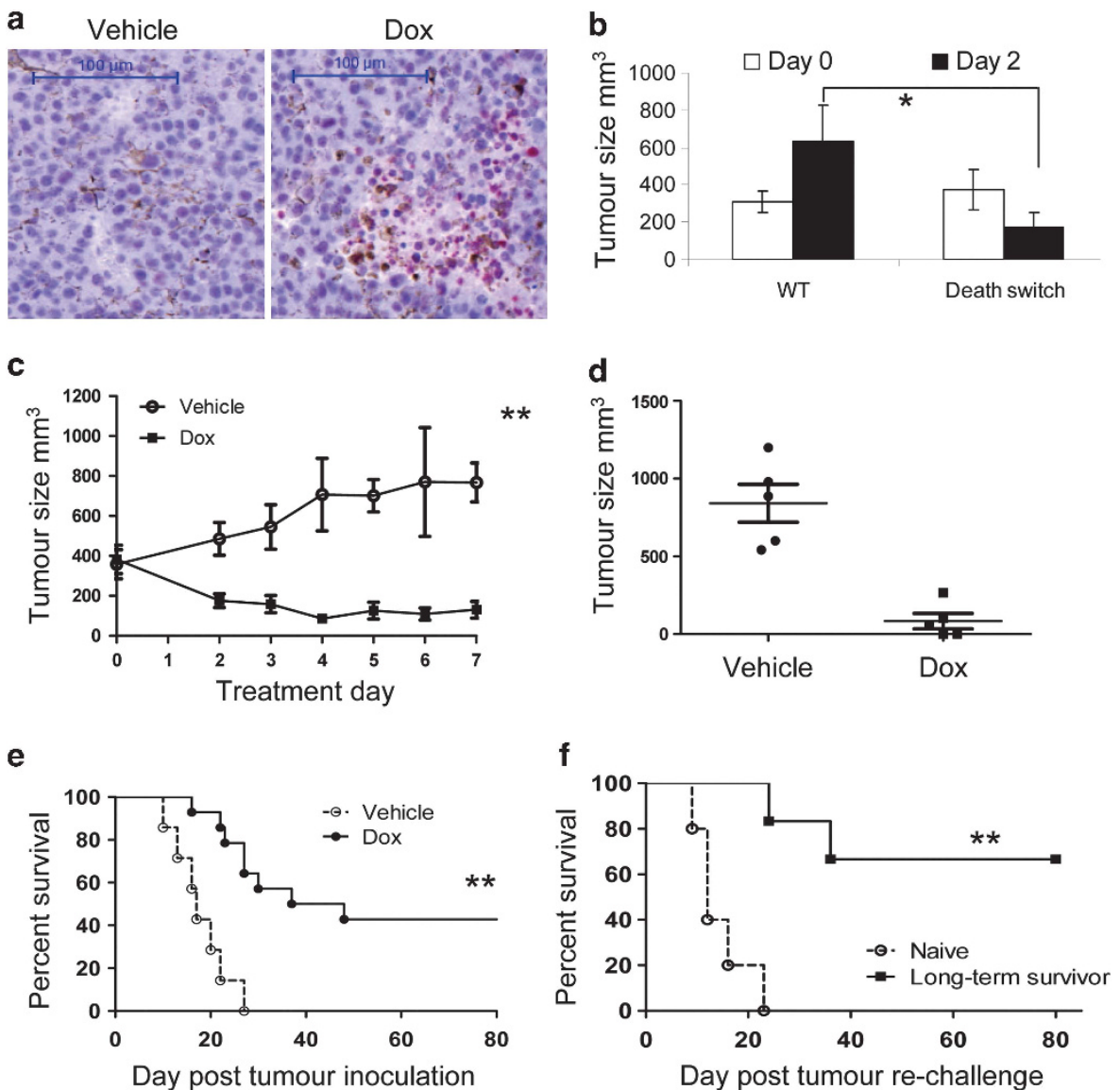

Figure 4 Doxycycline-regulated caspase-3 death switch model in vivo. C57BI/6 mice were inoculated with $1 \times 10^{6}$ death switch or parental cells s.c. on the right flank and the effect of death switch induction (dox, $0.2 \mathrm{ml}, 10 \mu \mathrm{g} / \mathrm{ml} \mathrm{p.0.)}$ ) on tumour growth measured over time. Dox was administered when the mean tumour volume reached $\sim 350 \mathrm{~mm}^{3}$. (a) Representative images showing immunohistochemical detection of cleaved PARP in death switch cells $24 \mathrm{~h}$ after administration of dox or vehicle control. Bars $=100 \mu \mathrm{m}$. (b) Reduction in tumour volume in mice bearing death switch versus parental tumour cells 2 days after a single administration of dox. ${ }^{*} P<0.05$. (c) Rate of tumour development over time during dox administration and (d) mean tumour volume 7 days after initial treatment in mice bearing death switch cells treated daily with dox or vehicle control for 7 days. Error bars represent mean \pm S.E.M. ${ }^{* *} P<0.01$. (e) Kaplan-Meier survival curve of C57B//6 mice inoculated with death switch cells and treated daily with vehicle control or dox for 7 days; and (f) long-term survivors ( $>60$ days after initial dox treatment) rechallenged with fresh death switch tumour cells $\left(1 \times 10^{6}\right.$ s.c., opposite flank) compared with naive controls. ${ }^{\star \star} P<0.01$. Data are representative of at least two individual experiments

nuclear localisation ${ }^{27}$ and may therefore influence HMGB1 mobility. Furthermore, it is unknown whether efflux of molecules during apoptosis is related purely to permeability changes as cells progress towards secondary necrosis or whether additional mechanisms operate. Hyperacetylation of HMGB1 can target the protein for exosomal secretion in activated monocytes ${ }^{28}$ and cells undergoing autophagy have also been shown to release HMGB1 even when plasma membrane integrity is maintained. ${ }^{29}$ Thus, it is possible that molecular signals released at progressive stages of the apoptotic pathway or from discrete cellular origins may be differentially modified and instigate distinct immune responses. A future goal will be to utilise the death switch system to address such issues, and further define the factors that contribute to immunogenic forms of apoptosis.

A key advantage of the death switch model is that it can be conditionally activated in vivo to induce apoptosis in developing tumours in the presence of an intact immune system. To date, investigations regarding the immunogenicity of apoptotic cancer cells have largely relied on prophylactic cellular vaccination strategies that have variably demonstrated apoptotic tumour cells to be immunogenic, ${ }^{15,30}$ weakly immunogenic ${ }^{31}$ or nonimmunogenic. ${ }^{32}$ This has led to a conflicting literature that may partly be a reflection of differences in experimental design or attributed to cells displaying a heterogeneous phenotype as they progress towards secondary apoptosis/necrosis during culture before inoculation. Furthermore, a major caveat of studies investigating the immunogenicity of apoptotic cell death using vaccination strategies is that by their very nature, such approaches are designed to induce responses in the absence of an established tumour microenvironment. The ability to induce death in the context of a developing tumour provides a more stringent setting for interrogating immunological responses in the presence of potential tumour immunesuppression networks. ${ }^{33}$

Surprisingly, tumour cells dying after induction of the apoptotic death switch were able to elicit a CD8 T celldependent anticancer immune response, leading to long-term eradication of tumour and protection against rechallenge. This was confirmed using immune-deficient animals and depleting $\mathrm{mAb}$ to eliminate CD8-positive T cells (although the 
a

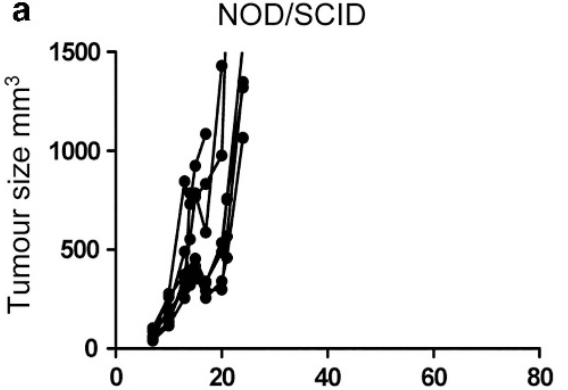

$\mathrm{C} 57 \mathrm{BI} / 6$

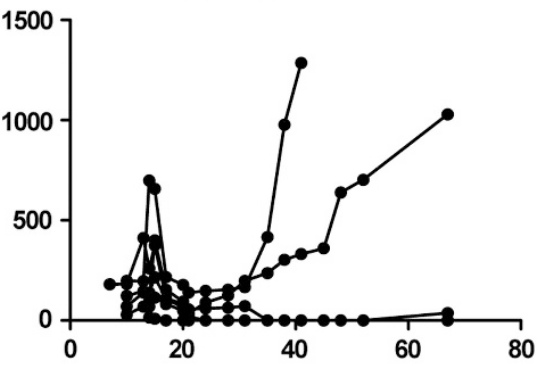

Day post tumour inoculation

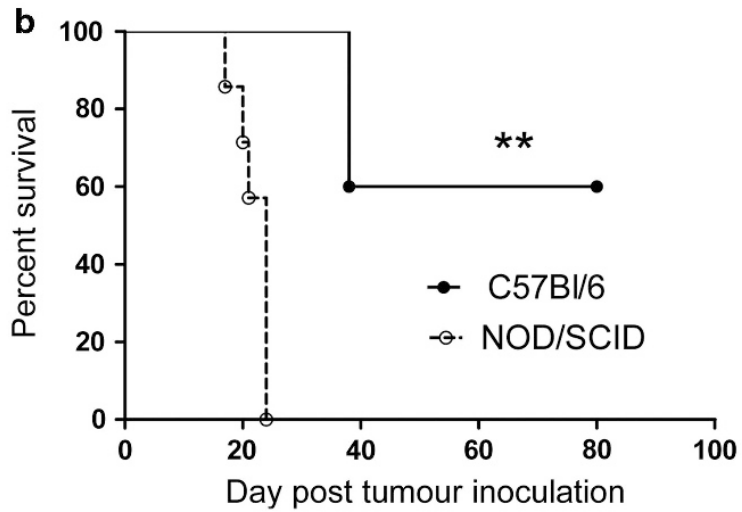

Figure 5 Caspase-3 death switch induction leads to improved survival only in the presence of an intact immune system. C57B//6 $(n=5)$ or NOD/SCID $(n=7)$ mice were inoculated with $1 \times 10^{6}$ death switch cells s.c. on the right flank and the effect of death switch induction (dox, $0.2 \mathrm{ml}, 10 \mu \mathrm{g} / \mathrm{ml}$ p.o.) on survival and tumour development measured over time. Dox was administered when the mean tumour volume reached $\sim 350 \mathrm{~mm}^{3}$. (a) Tumour volume over time, and (b) Kaplan-Meier survival plot of immunocompetent C57BI/6 or immunodeficient NOD/SCID mice treated daily with dox for 7 days. ${ }^{* *} P<0.01$. Data are representative of at least two individual experiments

activity of this mAb against other CD8-positive cells such as DCs may also potentially have an impact on immune activation). Although expression of revC3 leads to cell death accompanied by release of certain DAMPs and efficient uptake by DCs, at least in vitro, induction of robust antitumour immunity in vivo was unexpected. A possible explanation is that in the presence of high-rate synchronous apoptosis, as induced by the death switch, local phagocyte responses become subverted. Thus, although apoptotic cells are usually cleared rapidly by phagocytes such as macrophages in order to maintain tolerance and prevent unwanted inflammatory or autoimmune responses, ${ }^{34}$ in the presence of overwhelming amounts of simultaneous apoptosis, cells may escape clearance mechanisms and progress towards secondary necrosis, potentially enhancing recognition by DCs and subsequent cross-presentation of antigen to $\mathrm{T}$ cells. ${ }^{35}$ Whether this is sufficient to enhance immunogenicity remains to be determined as necrotic cell death is not always immunogenic, ${ }^{15}$ and studies have shown that signal transduction events induced by uptake/recognition of late apoptotic cells are indistinguishable from those induced by early apoptotic cells. ${ }^{36}$ The degree to which the amount of cell death within an established tumour affects immunogenicity and the role played by individual antigen-presenting cell subsets in priming the response can be addressed using titration of death switch cells with parental cells in vivo and phagocyte depletion systems, ${ }^{37,38}$ respectively.
To date, preclinical models for the targeted initiation of defined cell death pathways have been shown to favour the induction of necrosis ${ }^{39}$ or have only been utilised in vitro where the assessment of immunogenicity is severely limited. ${ }^{40}$ The death switch model enables the induction of apoptosis in vivo, thus providing a unique tool to help address the complex relationship between apoptotic tumour cell death, tumour microenvironment and subsequent host immune response. Understanding this interaction has potentially important implications for the therapeutic treatment of cancer. Models such as the death switch reported here, facilitating the induction of specific cell death pathways, will serve as important comparators for death induced by chemotherapeutic or other cytotoxic interventions, and will help to decipher why certain types of cell death are more immunogenic than others. Data obtained from the death switch model will also provide important clues as to how best enhance the generation and durability of antitumour immune responses in the clinic. One such approach is the combination of conventional apoptosis-inducing anticancer therapies with immunotherapeutic strategies that may cooperate in immune stimulation to enhance antitumour immune responses and long-term tumour clearance.

\section{Materials and Methods}

Cell lines and culture conditions. Murine melanoma cell line B16 and derivatives transfected with chicken ovalbumin (B16ova; kindly provided by 
a

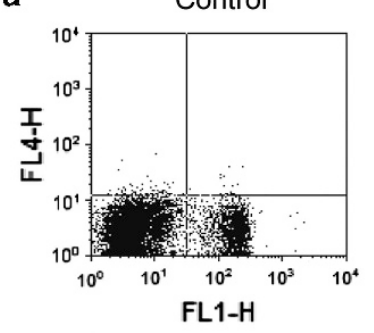

CD8 $\alpha$
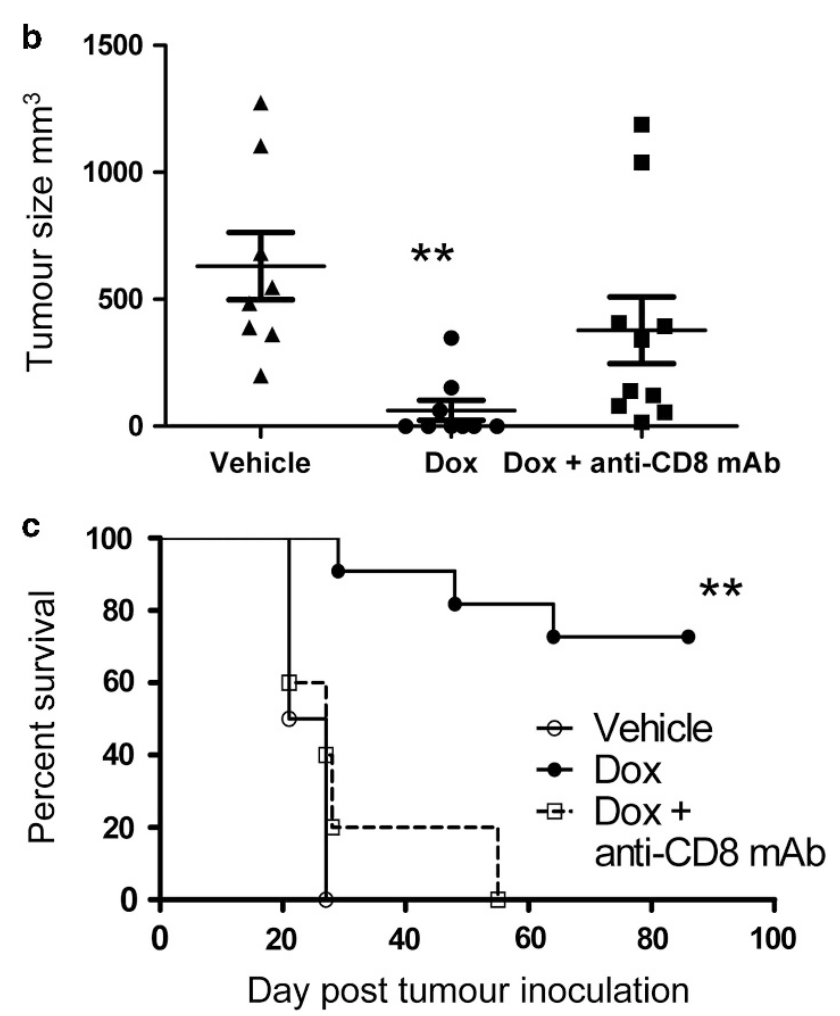

Figure 6 Depletion of CD8 T cells abrogates tumour regression after induction of the death switch. C57B//6 mice were inoculated with $1 \times 10^{6}$ death switch cells s.c. on the right flank and the effect of CD8 depletion on death switch induction (dox, $0.2 \mathrm{ml}, 10 \mu \mathrm{g} / \mathrm{ml}$ p.o.) on tumour growth measured over time. (a) Depletion of CD8-positive cells in peripheral blood was confirmed by flow cytometry. (b) Mean tumour volume 7 days after treatment, and (c) Kaplan-Meier survival curve survival plot of mice given vehicle control or dox in the presence or absence of CD8depleting antibody. ${ }^{* *} P<0.01$. Data are representative of at least two individual experiments

\begin{abstract}
A Melcher, Leeds, UK) were cultured in Roswell Park Memorial Institute (RPMI)1640 medium supplemented with $10 \%$ fetal calf serum (FCS) and $2 \mathrm{mM}$ L-glutamine (Invitrogen, Paisley, UK) in a humidified atmosphere at $37^{\circ} \mathrm{C}$ and $5 \% \mathrm{CO}_{2}$. B16 revC3 clones (death switch cells) were produced using a two-step process whereby Tet-on cells (transfected with $20 \mu \mathrm{g}$ module 1) were subsequently transfected with revC3, ${ }^{17}$ subcloned into the pTRE2hyg vector $(20 \mu \mathrm{g}$; Clontech, Oxford, UK) and cultured in the presence of $500 \mu \mathrm{g} / \mathrm{ml}$ hygromycin (Calbiochem, Nottingham, UK) to obtain individual colonies, as previously described. ${ }^{16}$
\end{abstract}

Cell death assay. Cells $\left(2 \times 10^{5}\right.$ cells/well) were pretreated, or not, with the cell-permeable pan-caspase inhibitor Q-VD-OPh ( $20 \mu \mathrm{M}$; Calbiochem) followed by addition of dox ( $2 \mu \mathrm{g} / \mathrm{ml}$; Sigma-Aldrich, Poole, UK) or vehicle control (water) for $6-24 \mathrm{~h}$ before analysis. Cells were harvested by trypsinisation and collected along with initial culture medium and wash buffer to ensure inclusion of detached cells. Cells were pelleted by centrifugation $(300 \times g$ for $5 \mathrm{~min}$ ) and re-suspended in annexin $\mathrm{V}$ binding buffer (10 mM HEPES, $140 \mathrm{mM} \mathrm{NaCl}$ and $2.5 \mathrm{mM} \mathrm{CaCl}_{2}$ ) containing $1 \mu \mathrm{g} / \mathrm{ml} \mathrm{CY5.5-annexin} \mathrm{V} \mathrm{(BD} \mathrm{Biosciences,} \mathrm{Oxford,} \mathrm{UK)} \mathrm{and} 10 \mu \mathrm{g} / \mathrm{ml}$ $\mathrm{PI}$ (Sigma-Aldrich) for $15 \mathrm{~min}$ at room temperature in the dark. Subsequently, cells were analysed by flow cytometry, using a FACSCalibur (BD Biosciences) operated by CellQuest software and at least 20000 events were collected per sample. Data were analysed using WinMDI software (Version 2.8, The Scripps Institute, La Jolla, CA, USA).

Western blotting. Cell lysates were prepared in lysis buffer (Cell Signaling, Hertfordshire, UK) supplemented with 1:100 protease cocktail inhibitor (SigmaAldrich). Protein concentrations were determined using the bicinchoninic acid assay (BCA) (Thermo Fisher Scientific, Runcorn, UK). Supernatants were collected and centrifuged for $5 \mathrm{~min}$ at $300 \times \mathrm{g}$, and the supernatant was transferred into a new vessel before use. Samples were separated by SDS-PAGE and transferred to polyvinylidene difluoride (PVDF) membrane using the Surelock Western Blotting Module (Invitrogen) according to the manufacturer's instructions. For protein detection, primary antibodies against cleaved caspase-3, (1:100) and cleaved PARP (1:1000) (Cell Signalling Technology, Hitchen, UK), HMGB1 (1: 1000; Abcam, Cambridge, UK) and HSP90 (1:1000; Stressgen, Cambridge, UK) were used; secondary antibodies were HRP-conjugated anti-rabbit or mouse IgG, 1:2500 (Dako, Ely, UK). Membranes were developed using ECL reagent (Perkin Elmer, Beaconsfield, UK) and visualised using an Intelligent Dark Box System (FUJIFILM, Tokyo, Japan).

Transmission electron microscopy. Samples were fixed overnight in a fixative containing $2 \%$ formaldehyde and $2.5 \%$ glutaraldehyde in $0.1 \mathrm{M}$ sodium cacodylate buffer $(\mathrm{pH} 7.4)$. Samples were washed $(0.1 \mathrm{M}$ sodium cacodylate buffer) followed by a 1 -h postfixation with reduced osmium in $0.1 \mathrm{M}$ sodium cacodylate buffer. Cell pellets were then incubated for $1 \mathrm{~h}$ with $1 \%$ tannic acid in $0.1 \mathrm{M}$ cacodylate buffer, washed and stained overnight $\left(4^{\circ} \mathrm{C}\right)$ with $1 \%$ uranyl acetate. Dehydration was performed in ethanol followed by propylene oxide. Samples were then infiltrated with LV resin (TAAB, Berkshire, UK) and polymerised at $60^{\circ} \mathrm{C}$ for $24 \mathrm{~h}$. Sections $(50-70 \mathrm{~nm})$ were imaged using a Tecnai 12 Biotwin electron microscope (FEl, Eindhoven, The Netherlands).

Immunohistochemistry. Sections were dewaxed and rehydrated and antigen retrieval was performed by heating the slides in $10 \mathrm{mM}$ citric acid buffer (pH 6.0) for 25 min. Casein solution (Vector Laboratories, Peterborough, UK) was applied for $30 \mathrm{~min}$ and removed before application of primary antibody (cleaved PARP, 1: 1000; Abcam) overnight $\left(4^{\circ} \mathrm{C}\right)$ followed by biotinylated anti-rabbit IgG (1:400; Dako). Subsequently, sections were incubated with alkaline phosphatase avidin-biotin complex and stained with Vector red substrate, containing levamisole (Vector Laboratories). Finally, slides were counterstained with haemotoxylin (Thermo Fisher Scientific) and dehydrated before mounting with a glass coverslip. High-resolution images were obtained using a Mirax digital slide scanning system (3DHistech, Budapest, Hungary).

Phagocytosis assay. Bone marrow DCs (BMDCs) were generated by stimulation of bone marrow leukocytes with granulocyte-macrophage colonystimulating factor (GM-CSF) according to Lutz et al. ${ }^{41}$ For the assay, target cells were labelled with the lipophilic red-dye, PKH-26 (Sigma-Aldrich), and then treated with dox or vehicle control for $24 \mathrm{~h}$. DCs were preincubated, or not, with cytochalasin $\mathrm{D}(10 \mu \mathrm{M})$ for $30 \mathrm{~min}$ before co-culture with target cells in a ratio of $1: 1$ in a 96-well plate for $24 \mathrm{~h}$. Samples were then harvested, stained with CD11cAPC (AbD Serotec, Kidlington, UK) to label DCs and analysed by flow cytometry. Phagocytosis was taken as the percentage of CD11c/PKH26 double-positive cells compared with CD11c-positive/PKH26-negative cells.

In vivo experiments. C57BI/6 and NOD/CB-17 SCID female mice (8-10 weeks old) were obtained from Harlan Laboratories (Bicester, UK). Cohorts of at least five mice were inoculated with $1 \times 10^{6} \mathrm{~B} 160 \mathrm{ovaRevC} 3$ tumour cells s.c. on day 0 . When the mean tumour volume reached $250-350 \mathrm{~mm}^{3}$, mice were treated with either dox ( $0.2 \mathrm{ml} \mathrm{p.0.,} 10 \mathrm{mg} / \mathrm{ml}$ ) or vehicle (water) by oral gavage once daily for up to 7 days. Tumour growth and survival were measured over time. For tumour rechallenge, mice received $1 \times 10^{6}$ tumour cells inoculated S.c. on the opposite flank to the primary inoculation. For CD8 T-cell depletion, mice received anti-CD8 mAb (YTS169, $0.5 \mathrm{mg}$, intraperitoneally) 1 day before initial dox 
administration, and subsequently every 3 days, for a total of 4 doses. Experiments were approved by the local ethical committee and performed under a United Kingdom Home Office licence.

Statistical analysis. Statistical significance was assessed by unpaired twotailed t-test, Mantel-Cox test or Mann-Whitney test as appropriate. Data are expressed as mean \pm S.E.M. $P<0.05$ was considered significant and $P<0.01$ as highly significant.

\section{Conflict of Interest}

The authors declare no conflict of interest.

Acknowledgements. We thank Professor Alan Melcher (University of Leeds UK) for the supply of B16ova cells, members of CEP for invaluable technical assistance and all members of the PICR flow cytometry and BRU core facilities for their help. The authors thank the staff in the EM facility in the Faculty of Life Sciences for their assistance, and the Wellcome Trust for equipment grant support to the EM facility. We also thank all members of the Targeted Therapy Group for helpful discussion and critical review of this manuscript. This work was supported by grants from Cancer Research UK (C431/A11335) and Leukaemia and Lymphoma Research.

1. Savill J, Fadok V. Corpse clearance defines the meaning of cell death. Nature 2000; 407 784-788.

2. Matzinger P. Tolerance, danger, and the extended family. Annu Rev Immunol 1994; 12 : 991-1045.

3. Rock KL, Lai JJ, Kono H. Innate and adaptive immune responses to cell death. Immuno Rev 2011; 243: 191-205.

4. Shi Y, Evans JE, Rock KL. Molecular identification of a danger signal that alerts the immune system to dying cells. Nature 2003; 425: 516-521.

5. Quintana FJ, Cohen IR. Heat shock proteins as endogenous adjuvants in sterile and septic inflammation. J Immunol 2005; 175: 2777-2782.

6. Zhang $Q$, Raoof $M$, Chen $Y$, Sumi $Y$, Sursal $T$, Junger $W$ et al. Circulating mitochondrial DAMPs cause inflammatory responses to injury. Nature 2010; 464: 104-107.

7. Galluzzi L, Vitale I, Abrams JM, Alnemri ES, Baehrecke EH, Blagosklonny MV et al. Molecular definitions of cell death subroutines: recommendations of the Nomenclature Committee on Cell Death 2012. Cell Death Differ 2012; 19: 107-120.

8. Okada H, Mak TW. Pathways of apoptotic and non-apoptotic death in tumour cells. Nat Rev Cancer 2004; 4: 592-603.

9. Michaud M, Martins I, Sukkurwala AQ, Adjemian S, Ma Y, Pellegatti P et al. Autophagydependent anticancer immune responses induced by chemotherapeutic agents in mice. Science 2011; 334: 1573-1577.

10. Ghiringhelli F, Apetoh L, Tesniere A, Aymeric L, Ma Y, Ortiz C et al. Activation of the NLRP3 inflammasome in dendritic cells induces IL-1beta-dependent adaptive immunity against tumors.. Nat Med 2009; 15: 1170-1178.

11. Kepp O, Tesniere A, Schlemmer F, Michaud M, Senovilla L, Zitvogel L et al. Immunogenic cell death modalities and their impact on cancer treatment. Apoptosis 2009; 14: 364-375

12. Obeid M, Tesniere A, Ghiringhelli F, Fimia GM, Apetoh L, Perfettini JL et al. Calreticulin exposure dictates the immunogenicity of cancer cell death. Nat Med 2007; 13: 54-61.

13. Apetoh L, Ghiringhelli F, Tesniere A, Obeid M, Ortiz C, Criollo A et al. Toll-like receptor 4-dependent contribution of the immune system to anticancer chemotherapy and radiotherapy. Nat Med 2007; 13: 1050-1059.

14. Obeid M, Panaretakis $T$, Joza N, Tufi R, Tesniere A, van Endert $P$ et al. Calreticulin exposure is required for the immunogenicity of gamma-irradiation and UVC light-induced apoptosis. Cell Death Differ 2007; 14: 1848-1850.

15. Casares N, Pequignot MO, Tesniere A, Ghiringhelli F, Roux S, Chaput N et al. Caspasedependent immunogenicity of doxorubicin-induced tumor cell death. J Exp Med 2005; 202 $1691-1701$

16. Welman A, Barraclough J, Dive C. Generation of cells expressing improved doxycycline regulated reverse transcriptional transactivator rtTA2S-M2. Nat Protoc 2006; 1: 803-811.

17. Srinivasula SM, Ahmad M, MacFarlane M, Luo Z, Huang Z, Fernandes-Alnemri T et al. Generation of constitutively active recombinant caspases -3 and -6 by rearrangement of their subunits. J Biol Chem 1998; 273: 10107-10111.
18. Panaretakis T, Kepp O, Brockmeier U, Tesniere A, Bjorklund AC, Chapman DC et al. Mechanisms of pre-apoptotic calreticulin exposure in immunogenic cell death. EMBO J 2009; 28: 578-590.

19. Earnshaw WC, Martins LM, Kaufmann SH. Mammalian caspases: structure, activation, substrates, and functions during apoptosis. Annu Rev Biochem 1999; 68: 383-424

20. MacCorkle RA, Freeman KW, Spencer DM. Synthetic activation of caspases: artificial death switches. Proc Natl Acad Sci USA 1998; 95: 3655-3660.

21. Scaffidi $\mathrm{P}$, Misteli T, Bianchi ME. Release of chromatin protein HMGB1 by necrotic cells triggers inflammation. Nature 2002; 418: 191-195

22. Basu S, Binder RJ, Suto R, Anderson KM, Srivastava PK. Necrotic but not apoptotic cell death releases heat shock proteins, which deliver a partial maturation signal to dendritic cells and activate the NF-kappa B pathway. Int Immunol 2000; 12: 1539-1546.

23. Bell CW, Jiang W, Reich CF 3rd, Pisetsky DS. The extracellular release of HMGB1 during apoptotic cell death. Am J Physiol Cell Physiol 2006; 291: C1318-C1325.

24. Martins I, Tesniere A, Kepp O, Michaud M, Schlemmer F, Senovilla L et al. Chemotherapy induces ATP release from tumor cells. Cell Cycle 2009; 8: 3723-3728.

25. Choi JJ, Reich CF 3rd, Pisetsky DS. Release of DNA from dead and dying lymphocyte and monocyte cell lines in vitro. Scand J Immunol 2004; 60: 159-166.

26. Kazama H, Ricci JE, Herndon JM, Hoppe G, Green DR, Ferguson TA. Induction of immunological tolerance by apoptotic cells requires caspase-dependent oxidation of high-mobility group box-1 protein. Immunity 2008; 29: 21-32.

27. Hoppe G, Talcott KE, Bhattacharya SK, Crabb JW, Sears JE. Molecular basis for the redox control of nuclear transport of the structural chromatin protein Hmgb1. Exp Cell Res 2006; 312: $3526-3538$

28. Bonaldi T, Talamo F, Scaffidi P, Ferrera D, Porto A, Bachi A et al. Monocytic cells hyperacetylate chromatin protein HMGB1 to redirect it towards secretion. EMBO J 2003; 22: $5551-5560$.

29. Thorburn J, Horita H, Redzic J, Hansen K, Frankel AE, Thorburn A. Autophagy regulates selective HMGB1 release in tumor cells that are destined to die. Cell Death Differ 2009; 16 : 175-183.

30. Scheffer SR, Nave H, Korangy F, Schlote K, Pabst R, Jaffee EM et al. Apoptotic, but not necrotic, tumor cell vaccines induce a potent immune response in vivo. Int J Cancer 2003; 103: 205-211.

31. Ronchetti A, Rovere P, lezzi G, Galati G, Heltai S, Protti MP et al. Immunogenicity of apoptotic cells in vivo: role of antigen load, antigen-presenting cells, and cytokines. J Immunol 1999; 163: 130-136.

32. Sauter B, Albert ML, Francisco L, Larsson M, Somersan S, Bhardwaj N. Consequences of cell death: exposure to necrotic tumor cells, but not primary tissue cells or apoptotic cells, induces the maturation of immunostimulatory dendritic cells. J Exp Med 2000; 191 : 423-434.

33. Zou W. Immunosuppressive networks in the tumour environment and their therapeutic relevance. Nat Rev Cancer 2005; 5: 263-274.

34. Gregory CD, Devitt A. The macrophage and the apoptotic cell: an innate immune interaction viewed simplistically? Immunology 2004; 113: 1-14.

35. Rovere P, Sabbadini MG, Vallinoto C, Fascio U, Zimmermann VS, Bondanza A et al. Delayed clearance of apoptotic lymphoma cells allows cross-presentation of intracellular antigens by mature dendritic cells. J Leukoc Biol 1999; 66: 345-349.

36. Patel VA, Longacre A, Hsiao K, Fan H, Meng F, Mitchell JE et al. Apoptotic cells, at all stages of the death process, trigger characteristic signaling events that are divergent from and dominant over those triggered by necrotic cells: implications for the delayed clearance model of autoimmunity. J Biol Chem 2006; 281: 4663-4670.

37. van Rooijen N, van Kesteren-Hendrikx E. "In vivo" depletion of macrophages by liposomemediated "suicide". Methods Enzymol 2003; 373: 3-16.

38. Jung S, Unutmaz D, Wong P, Sano G, De los Santos K, Sparwasser T et al. In vivo depletion of $\mathrm{CD} 11 \mathrm{c}+$ dendritic cells abrogates priming of $\mathrm{CD} 8+\mathrm{T}$ cells by exogenous cell-associated antigens. Immunity 2002; 17: 211-220.

39. Vile RG, Castleden S, Marshall J, Camplejohn R, Upton C, Chong H. Generation of an anti-tumour immune response in a non-immunogenic tumour: HSVtk killing in vivo stimulates a mononuclear cell infiltrate and a Th1-like profile of intratumoural cytokine expression. Int J Cancer 1997; 71: 267-274.

40. Lohmann C, Muschaweckh A, Kirschnek S, Jennen L, Wagner H, Hacker G. Induction of tumor cell apoptosis or necrosis by conditional expression of cell death proteins: analysis of cell death pathways and in vitro immune stimulatory potential. J Immunol 2009; 182: $4538-4546$.

41. Lutz MB, Kukutsch N, Ogilvie AL, Rossner S, Koch F, Romani N et al. An advanced culture method for generating large quantities of highly pure dendritic cells from mouse bone marrow. J Immunol Methods 1999; 223: 77-92. 